\title{
ON SOME EQUALITIES AND INEQUALITIES \\ OF FUSION FRAME IN HILBERT $C^{*}$-MODULES
}

\author{
DONGWEI Li, Jinsong LENG AND TINGZHU HuANG
}

\begin{abstract}
In this paper, we establish some new identities and inequalities for fusion frames with a scalar in Hilbert $C^{*}$-modules. Our results are more general than those previously obtained by Balan et al. for Hilbert space frames. It is shown that the results we obtained can immediately lead to the existing corresponding results when taking suitable scalar. Moreover, we give some double inequalities for fusion frames in Hilbert $C^{*}$-modules.
\end{abstract}

Mathematics subject classification (2010): 46L99, 42C15, 46H25.

Keywords and phrases: Fusion frames, Hilbert $C^{*}$-modules, inequality, operator.

\section{REFERENCES}

[1] Morteza Mirzaee AZAndaryani, Erasures and perturbations of $g$-frames and fusion frames in Hilbert C-modules, International Journal of Wavelets, Multiresolution and Information Processing, 14(01):1650003, 2016.

[2] M. AZHini AND N. HADDADZADEH, Fusion frames in Hilbert modules over pro-C*-algebras, International Journal of Industrial Mathematics, 5(2):109-118, 2013.

[3] Radu Balan, Pete CasazZa And Dan Edidin, On signal reconstruction without phase, Applied and Computational Harmonic Analysis, 20(3):345-356, 2006.

[4] Radu Balan, Peter Casazza, Dan Edidin and Gitta Kutyniok, A new identity for Parseval frames, Proceedings of the American Mathematical Society, 135(4):1007-1015, 2007.

[5] Wei Chen, Miguel RD Rodrigues and Ian J Wassell, Projection design for statistical compressive sensing: A tight frame based approach, IEEE Transactions on signal processing, 61(8):20162029, 2013.

[6] Ole Christensen, An introduction to frames and Riesz bases, volume 7. Springer, 2003.

[7] Richard J DufFin ANd Albert C SChAEFFER, A class of nonharmonic Fourier series, Transactions of the American Mathematical Society, 72(2):341-366, 1952.

[8] Michael Frank AND David R LARSON, Frames in Hilbert $C^{*}$-modules and $C^{*}$-algebras, Operator Theory, 48(2):273-314, 2002.

[9] P. GĂVRUŢA, On some identities and inequalities for frames in Hilbert spaces, Journal of mathematical analysis and applications, 321(1):469-478, 2006.

[10] P. Găvruţa, On the duality of fusion frames, Journal of Mathematical Analysis and Applications, 333(2):871-879, 2007.

[11] Vivek K Goyal, Jelena Kovačević and Jonathan A Kelner, Quantized frame expansions with erasures, Applied and Computational Harmonic Analysis, 10(3):203-233, 2001.

[12] Amir KhosraVi And M MirZAeE AZANDARYANi, Fusion frames and g-frames in tensor product and direct sum of Hilbert spaces, Applicable Analysis and Discrete Mathematics, pages 287-303, 2012.

[13] Amir Khosravi And Behrooz Khosravi, Fusion frames and $g$-frames in Hilbert $C^{*}$-modules, International Journal of Wavelets, Multiresolution and Information Processing, 6(03):433-446, 2008.

[14] Jelena Kovacevic, Pier Luigi Dragotti and Vivek K Goyal, Filter bank frame expansions with erasures, IEEE Transactions on Information Theory, 48(6):1439-1450, 2002.

[15] Jinsong Leng And Deguang Han, Optimal dual frames for erasures II, Linear Algebra and its Applications, 435(6):1464-1472, 2011. 
[16] Jinsong Leng, Deguang Han And Tingzhu Huang, Optimal dual frames for communication coding with probabilistic erasures, IEEE transactions on signal processing, 59(11):5380-5389, 2011.

[17] Dongwei Li And Jinsong Leng, On some new inequalities for fusion frames in Hilbert spaces, Math. Inequal. Appl, 20:889-900, 2017.

[18] Dongwei Li AND Jinsong Leng, On some new inequalities for continuous fusion frames in Hilbert spaces, Mediterranean Journal of Mathematics, 15(4):173, 2018.

[19] Dongwei Li, Jinsong Leng, Tingzhu Huang And Yuxiang Xu, Some equalities and inequalities for probabilistic frames, Journal of Inequalities and Applications, 2016(1):245, 2016.

[20] Anirudha Poria, Some identities and inequalities for Hilbert-Schmidt frames, Mediterranean Journal of Mathematics, 14(2):59, 2017.

[21] Zhong-Qi Xiang, New inequalities for g-frames in Hilbert $c^{*}$-modules, Journal of Mathematical Inequalities, 10(3):889-897, 2016.

[22] Xiang-Chun Xiao And XiaO-Ming Zeng, Some properties of g-frames in Hilbert $C$-modules, Journal of Mathematical Analysis and Applications, 363(2):399-408, 2010.

[23] XH YANG AND DF LI, Some new equalities and inequalities for $g$-frames and their dual frames, Acta Mathematica Sinica, Chinese Series, 52(5):1033-1040, 2009. 